\title{
miR-203 enhances chemosensitivity to 5-fluorouracil by targeting thymidylate synthase in colorectal cancer
}

\author{
TAO $\mathrm{LI}^{1}$, FENG GAO ${ }^{2}$ and XI-PENG ZHANG $^{1}$ \\ ${ }^{1}$ Colorectal Center, Tianjin Union Medical Center, Tianjin 300121; ${ }^{2}$ Department of General Surgery, \\ Qingdao Hiser Medical Center, Qingdao, Shandong 266033, P.R. China
}

Received June 27, 2014; Accepted September 2, 2014

DOI: $10.3892 /$ or.2014.3646

\begin{abstract}
MicroRNAs (miRNAs) are a conserved class of small non-coding RNAs that play important roles in diverse biological processes, including chemoresistance. However, the molecular mechanism as to how miR-203 modulates the chemosensitivity to 5-fluorouracil (5-FU) in colorectal cancer is poorly known. In the present study, we found that miR-203 was downregulated in the 5-FU-resistant cell line $\mathrm{LoVo} / 5-\mathrm{Fu}$, and was inversely correlated with the extent of 5-FU chemoresistance. Cytotoxicity assay showed that the inhibition of miR-203 expression enhanced 5-FU chemoresistance in colorectal cancer cells, while miR-203 overexpression increased 5-FU chemosensitivity. We then validated that thymidylate synthase (TYMS) was a direct target of miR-203 and miR-203 suppressed TYMS protein levels. Silencing of TYMS enhanced 5-FU chemosensitivity, similar to the roles of miR-203. Finally, we discovered that miR-203 increased the inhibitory effects of 5-FU on tumor growth in vivo. Overall, our data indicate that miR-203 enhances 5-FU chemosensitivity via the downregulation of TYMS in colorectal cancer and provide important insight into the mechanism of 5-FU resistance in colorectal cancer patients. More important, the present study suggests that miR-203 has the potential as a therapeutic strategy for 5-FU-resistant colorectal cancer.
\end{abstract}

\section{Introduction}

Colorectal cancer is the third leading cause of cancer-related deaths worldwide, and the 5-year survival rate is mostly dependent on the cancer stage, resulting in a survival rate ranging from 10 to $95 \%$ (1). If patients are not diagnosed in the early stage, this malignancy seriously threatens patient survival. 5-Fluorouracil (5-FU) is a widely used therapeutic

Correspondence to: Dr Feng Gao, Department of General Surgery, Qingdao Hiser Medical Center, Qingdao, Shandong 266033, P.R. China E-mail: gaofeng0101_a@163.com

Key words: microRNAs, miR-203, thymidylate synthase, 5-fluorouracil, chemosensitivity agent for treating advanced colorectal cancer (2). However, some patients develop 5-FU resistance which is a major obstacle to effective therapy. Therefore, there is urgent need to elucidate the molecular mechanism underlying the 5-FU resistance in colorectal cancer cells and develop novel diagnostic biomarkers for 5-FU resistance that will facilitate the development of therapeutic strategies for colorectal cancer patients.

Thymidylate synthase (TYMS) is a key therapeutic target of 5-FU and serves as a predictive biomarker of the cellular response to 5-FU treatment. The antitumor effect of 5-FU was found to be promoted by rosemary extract via the downregulation of TYMS in 5-FU-resistant colorectal cancer cells (3). One study demonstrated that metastatic colorectal cancer that did not response to 5-FU treatment had a high TYMS level (4). High TYMS expression induces 5-FU resistance in human colon cancer cells that are continuously exposed to 5-FU (5). In addition, TYMS is associated with 5-FU resistance of colon and gastric cancers $(6,7)$.

Emerging evidence demonstrates that $\sim 60 \%$ of proteincoding genes are controlled by microRNAs (miRNAs) (8), which are a conserved class of small ( $22 \mathrm{nt})$ non-coding RNA molecules and that target mRNA cleavage or translational repression via binding to the sequences on the $3^{\prime}$ untranslated region (3'UTR) of target mRNAs with complete or incomplete complementarity (9). miRNAs play important roles in diverse biological processes, such as cell proliferation, cell death and developmental timing (10). Evidence indicates that aberrant miRNA expression is linked to the initiation and development of diverse cancers, as well as cancer cell resistance to therapeutic agents (11-13). For example, miR-129 increases cell sensitivity to 5 -FU by targeting $\mathrm{Bcl}-2$ in colorectal cancer (14). miR-433 enhances HeLa cellular sensitivity to 5-FU by downregulating TYMS (15), a first report concerning the regulation of TYMS by miRNAs in the cell response to 5-FU. However, the regulation of cell resistance to 5-FU by miR-203 in colorectal cancer remains known.

In the present study, we found that miR-203 was downregulated in 5-FU-resistant cell line LoVo/5-Fu compared with LoVo cells using miRNA microarray. miR-203 expression was inversely correlated with the extent of 5-FU resistance. miR-203 inhibition enhanced cell resistance to 5-FU, while miR-203 overexpression increased 5-FU sensitivity. TYMS was validated as a direct target of miR-203 and TYMS knockdown resulted in similar effects on the cellular response to 
5-FU as that of miR-203 overexpression. Finally, we found that the inhibitory effect of 5-FU on colorectal cancer growth was enhanced by miR-203 overexpression.

\section{Materials and methods}

Cell culture. Human normal colorectal mucosa FHC cells and colorectal cancer cell lines HCT-116, Caco2 and SW480 were cultured in DMEM. HT29 and DLD1 cells were cultured in RPMI-1640, and LoVo cells were cultured in F12K medium (Gibco, Life Technologies, USA). All medium was supplemented with $10 \%$ fetal bovine serum (FBS) (Gibco, Life Technologies) and $1 \%$ antibiotic solution (100 U/ml penicillin and $100 \mu \mathrm{g} / \mathrm{ml}$ streptomycin) (Sigma, USA). The cells were maintained in a humidified incubator (Thermo Fisher, USA) at $37^{\circ} \mathrm{C}$ with $5 \% \mathrm{CO}_{2}$. The 5-FU highly resistant cell line $\mathrm{LoVo} / 5-\mathrm{Fu}(\mathrm{HR})$ was acquired by treating LoVo cells with 5-FU (Sigma) ranging from a concentration of 0.1 to $2 \mu \mathrm{g} / 1$ for $\sim 11$ months. The middle resistant cell line LoVo/5-Fu (MR) was acquired after $\sim 6$ months of treatment.

RNA isolation and $q R T-P C R$. Total RNAs (including miRNAs) were extracted from cells or tumor tissues using TRIzol reagent (Invitrogen, USA) according to the manufacturer's instructions. After the RNA concentration was measured, $500 \mathrm{ng}$ of RNA was reverse transcribed using a specific miR-203 primer by M-MLV reverse transcriptase (Takara, Japan). qRT-PCR was performed using SYBR-Green PCR Master Mix and specific miR-203 primers on an ABI 7300 real-time PCR system. Small nuclear RNA U6 (U6 snRNA) was used as an internal control to normalize miR-203 expression. The primers for reverse transcription and PCR were as follows: miR-203 reverse transcript primer, 5'-CTCAACTGG TGTCGTGGAGTCGGCAATTCAGTTGAGCTAGTGGT-3'; miR-203 forward primer, 5'-ACACTCCAGCTGGGGTGAA ATGTTTA-3' and miR-203 reverse primer, 5'-TGGTGTCGT GGAGTCG-3'; U6 forward, CTCGCTTCGGCAGCACA3' and U6 reverse, 5'AACGCTTCACGAATTTGCGT-3'.

Western blotting. After transfection with miR-203 ASO or miR-203 mimics or siRNA against TYMS or scrambled controls for $\sim 48 \mathrm{~h}$, colorectal cancer cells were harvested and lysed using RIPA buffer (50 mM Tris-HCl, pH 8.8, $150 \mathrm{mM}$ $\mathrm{NaCl}, 1 \% \mathrm{NP}-40,1 \%$ sodium deoxycholate, $0.1 \%$ SDS). Proteins were also extracted from the tumor tissues using RIPA according to the manufacturer's protocols. Proteins $(30 \mu \mathrm{g})$ were separated on a SDS-PAGE gel and then transferred to a polyvinylidene difluoride (PVDF) membrane, followed by incubation with 5\% milk. The membrane was incubated in the mouse monoclonal to TYMS antibody (used as a primary antibody) (1:1,000; Abcam, USA) overnight at $4^{\circ} \mathrm{C}$, and subsequently in the HRP-conjugated goat anti-mouse antibody (used as a secondary antibody) for $1.5 \mathrm{~h}$. Finally, the protein signals were detected using enhanced chemiluminescence (ECL) according to the manufacturer's instructions. GAPDH was used as a loading control to normalize the expression of TYMS

Luciferase assay. The 3'UTR of TYMS containing the binding site for miR-203 was amplified and cloned in the
Table I. The $\mathrm{IC}_{50}$ values of $5-\mathrm{FU}$ and CDDP in LoVo and 5-Fu-resistant LoVo cell lines.

\begin{tabular}{lcc}
\hline Cell lines & $\mathrm{IC}_{50}(\mu \mathrm{mol} / \mathrm{l})$ & $\mathrm{IC}_{50}(\mu \mathrm{mol} / \mathrm{l})$ \\
\hline LoVo & $5-\mathrm{FU}$ & $\mathrm{CDDP}$ \\
LoVo/5-FU (MR) & $1.62 \pm 0.14$ & $4.13 \pm 0.62$ \\
LoVo/5-FU (HR) & $4.28 \pm 0.47^{\mathrm{a}}$ & $43.91 \pm 0.51^{\mathrm{ns}}$ \\
\hline
\end{tabular}

${ }^{\mathrm{a}} \mathrm{p}<0.05 . \mathrm{LoVo} / 5-\mathrm{Fu}(\mathrm{MR})$ vs. LoVo group. ${ }^{\mathrm{b}} \mathrm{p}<0.05 . \mathrm{LoVo} / 5-\mathrm{Fu}(\mathrm{HR})$ vs. LoVo group. ${ }^{c} \mathrm{p}<0.05$. LoVo/5-Fu (HR) vs. LoVo/5-Fu (MR) group. ${ }^{n s}$ Non-significant between the two groups. 5-FU, 5-fluorouracil.

downstream region of a luciferase coding gene. Several base mutations within the binding site were generated using a QuikChange ${ }^{\circledR}$ site-directed mutagenesis kit (Stratagene, USA). Colorectal cancer cells were co-transfected with either $40 \mathrm{nM}$ miR-203 ASO or miR-203 mimics and $200 \mathrm{ng}$ of wild-type or mutant TYMS 3'UTR. After transfection for $48 \mathrm{~h}$, cells were harvested to analyze the luciferase intensity using a Dual-Glo luciferase assay (Promega, USA) according to the manufacturer's instructions. Renilla luciferase intensity was used to normalize the firefly luciferase intensity.

5-FU treatment and cytotoxicity assay. Colorectal cancer cells transfected with miR-203 ASO or miR-203 mimics or scrambled controls were seeded in 96-well plates with 5,000 cells/well. Twenty-four hours after cell adhesion, an additional $100 \mu \mathrm{l}$ of 5-FU solution was added for $72 \mathrm{~h}$. The 5-FU concentration varied from 0.2 to $3.2 \mu \mathrm{M}$ in the LoVo cells, from 2 to $32 \mu \mathrm{M}$ in the SW480, HT29 and LoVo/5-Fu (HR) cells, and from 1 to $16 \mu \mathrm{M}$ in the Caco2 cells. The cytotoxicity was measured using a MTT assay according to the manufacturer's information.

Xenograft assay. The mouse experiments were approved by the Institutional Animal Care and Use Committee of Tianjin Union Medical Center, and performed under specific pathogen-free conditions. LoVo $\left(1 \times 10^{6}\right)$ cells were suspended in $100 \mu \mathrm{l}$ of phosphate-buffered saline and subcutaneously injected into the flank of 4-5 week NOD/SCID mice. On day 12 when the tumor size reached $\sim 100 \mathrm{~mm}^{3}$, the mice were randomly separated into four groups and subsequently injected with the miR-203-negative control or miR-203 or 5-FU alone or 5-FU and miR-203 together on day 12, 15, 18, 21 and 25. Tumor volume was examined by measuring the length $(\mathrm{L})$ and width (W) with calipers and was calculated with the formula $\left(\mathrm{LxW}^{2}\right) / 2$.

Statistical analysis. All experiments were confirmed at least three times. The data are shown as means \pm standard deviation (SD) and one representative data value is shown in the present study. The statistical significance was evaluated using two-tailed Student's t-test between two groups and analyzed using GraphPad Prism software. A difference was considered statistically significant at a value of $p<0.05$. 


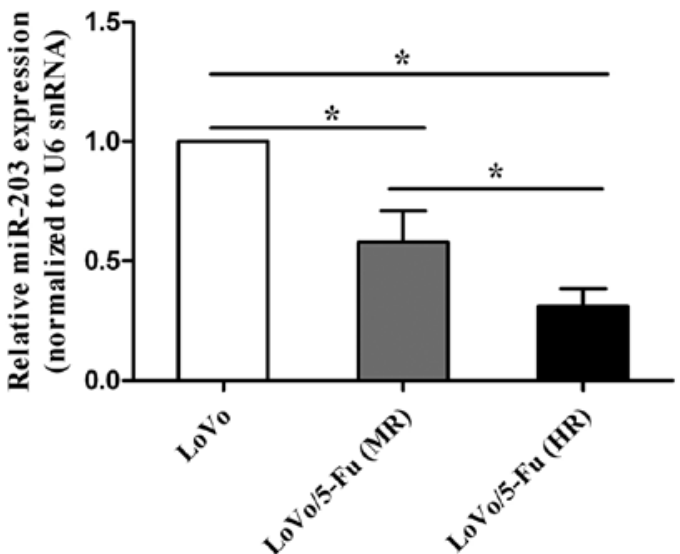

Figure 1. miR-203 is downregulated in 5-FU-resistant colorectal cancer cells. miR-203 expression was quantified by qRT-PCR in LoVo, LoVo/5-Fu (MR) and $\mathrm{LoVo} / 5-\mathrm{Fu}(\mathrm{HR})$ cells. U6 snRNA served as an internal control for normalization. ${ }^{*} \mathrm{p}<0.05$. Error bars represented the SD of samples in triplicate. 5-FU, 5-fluorouracil.

\section{Results}

miR-203 is downregulated in 5-FU resistant cells. To investigate the differentially expressed miRNAs in 5-FU-resistant cells, we performed miRNA microarray between the LoVo and LoVo/5-Fu cells and found that miR-203 was expressed to the lesser degree in the $\mathrm{LoVo} / 5-\mathrm{Fu}$ cells. Cytotoxicity assay indicated that $\mathrm{LoVo} / 5-\mathrm{Fu}(\mathrm{MR})$ and $\mathrm{LoVo} / 5-\mathrm{Fu}(\mathrm{HR})$ cells had a higher $\mathrm{IC}_{50}$ value for 5-FU than that of the LoVo cells (Table I), while there was no significant difference in the $\mathrm{IC}_{50}$ value for CDDP among these cell lines. Based on the miRNA microarray data, miR-203 expression was confirmed using qRT-PCR in the LoVo, LoVo/5-Fu (MR) and LoVo/5-Fu (HR) cell lines (Fig. 1). Taken together, these data indicate that miR-203 expression may be negatively correlated with the $\mathrm{IC}_{50}$ value of 5-FU, suggesting that miR-203 is closely related to 5-FU chemosensitivity.

miR-203 inhibition enhances 5-FU chemoresistance in colorectal cancer cells. To determine the effect of miR-203 on the cell sensitivity to 5-FU in colorectal cancer, we first detected miR-203 expression in a series of colorectal cancer cell lines. qRT-PCR assay showed that miR-203 was upregulated in most of the colorectal cancer cell lines, except Caco2 cells (Fig. 2A). Second, we suppressed endogenous miR-203 expression in LoVo and SW480 cells by transient transfection with miR-203 ASO or scrambled control. We found that miR-203 was significantly reduced in the miR-203 ASO-transfected cells (Fig. 2B). Finally, cytotoxicity assay indicated that miR-203 inhibition resulted in an increase in the survival rate of LoVo cells, which implies that miR-203 inhibition enhances the chemoresistance to 5-FU (Fig. 2C). Similar results were observed in the SW480 cells (Fig. 2D).
$\mathbf{A}$
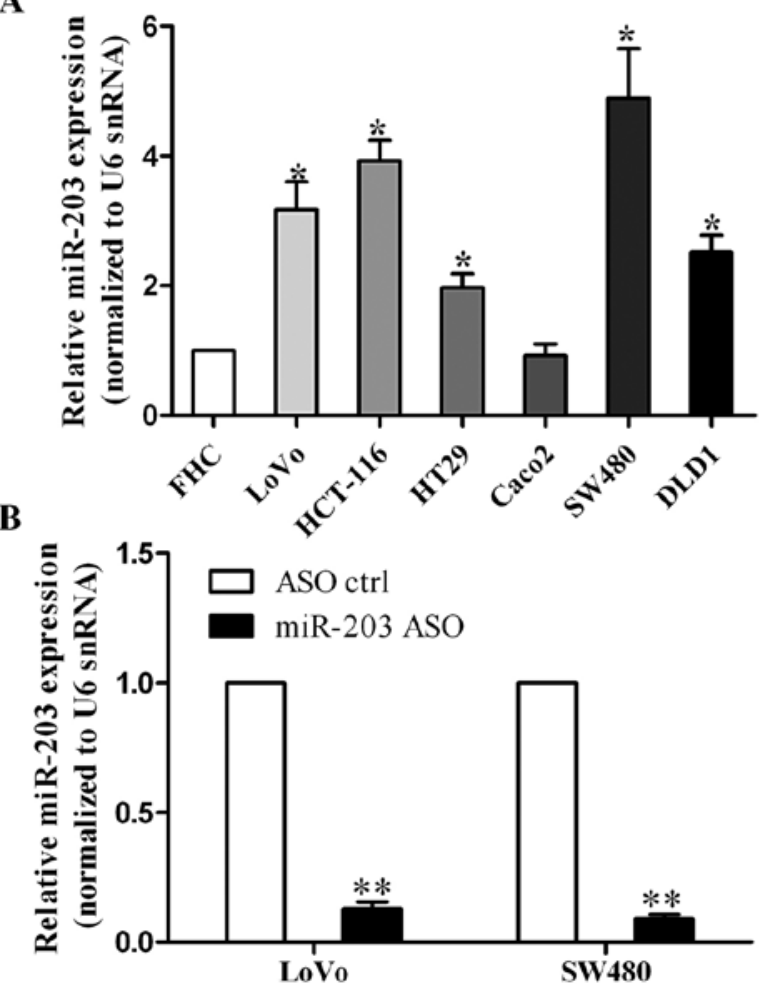

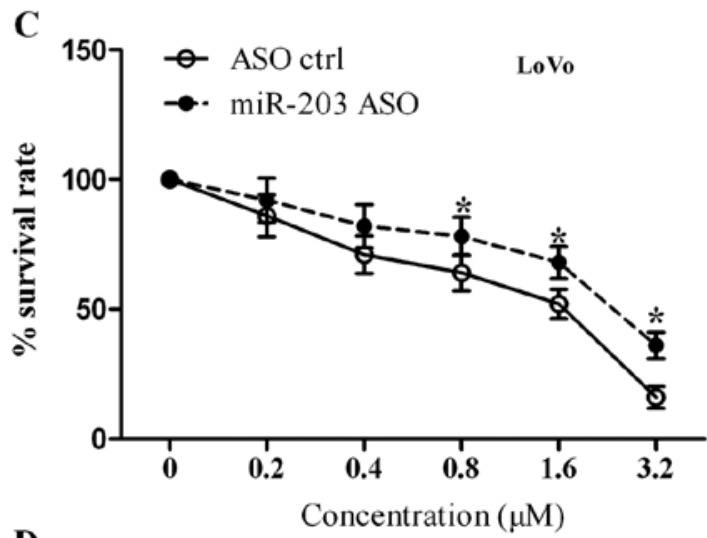

D

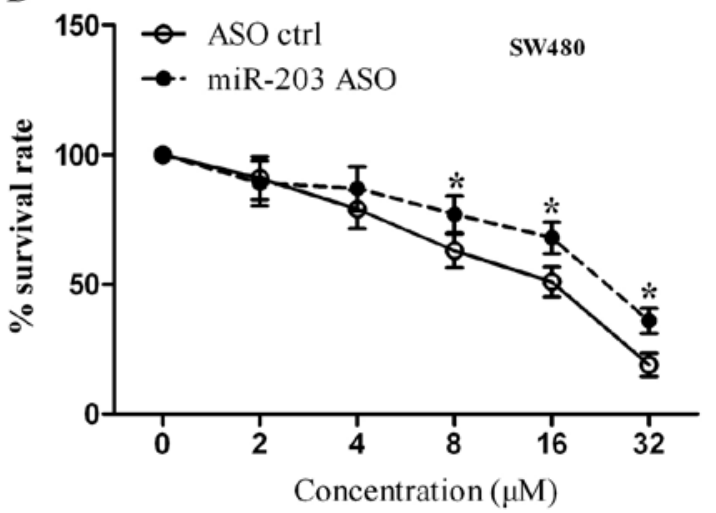

Figure 2. Inhibition of miR-203 enhances the chemoresistance to 5-FU in colorectal cancer cells. (A) miR-203 expression was determined by qRT-PCR in normal colorectal mucosa FHC cells and a series of colorectal cancer cell lines. (B) miR-203 expression was analyzed by qRT-PCR in LoVo and SW480 cells transfected with miR-203 ASO or scrambled ASO control. U6 snRNA served as an internal control for normalization. * $\mathrm{p}<0.05$. ${ }^{* *} \mathrm{p}<0.01$. Error bars represent the SD of samples in triplicate. (C and D) LoVo and SW480 cells transfected with miR-203 ASO or control were exposed to 5-FU treatment at different concentrations and 5-FU chemosensitivity was determined by MTT assay. "p<0.05. Error bars represent the SD of samples in triplicate. 5-FU, 5-fluorouracil. 
A

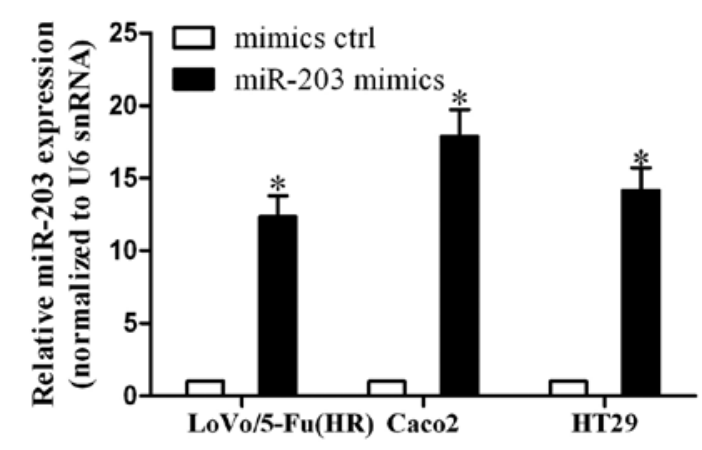

C

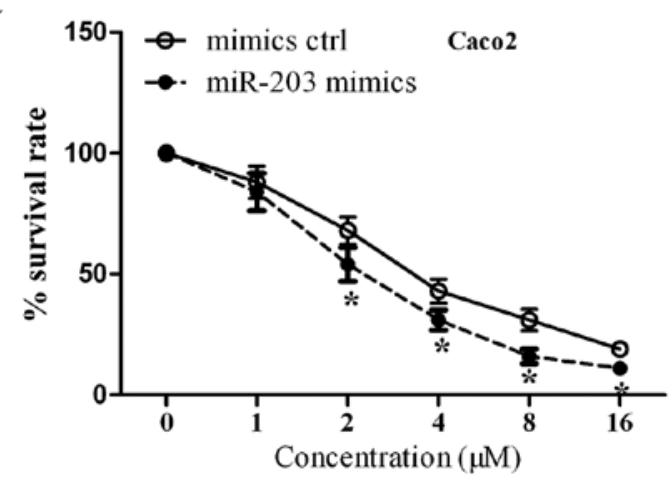

B

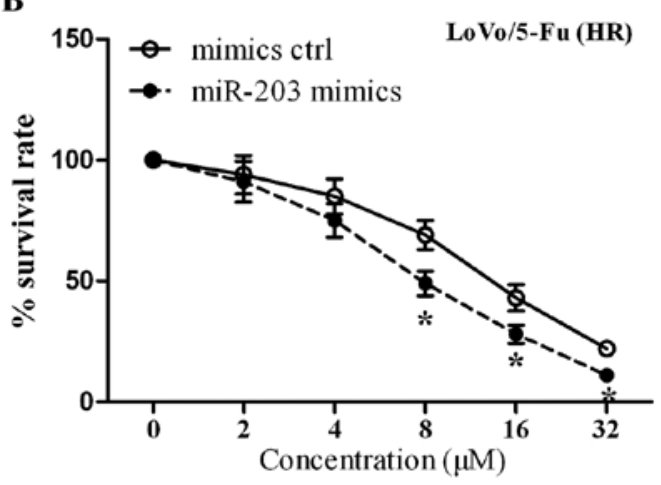

D

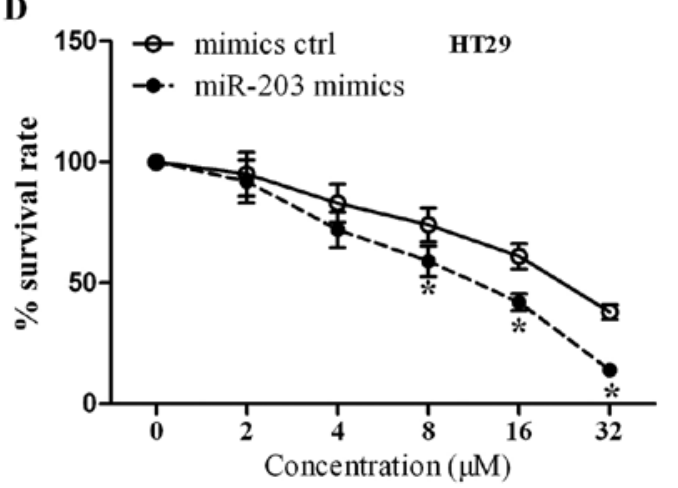

Figure 3. miR-203 overexpression enhances the chemosensitivity to 5-FU in LoVo/5-Fu (HR) cells and colorectal cancer cell lines. (A) miR-203 expression was analyzed by qRT-PCR in LoVo/5-Fu (HR), Caco2 and HT29 cells transfected with miR-203 mimics or mimic control. U6 snRNA served as an internal control for normalization. (B) LoVo/5-Fu (HR), (C) Caco2 and (D) HT29 cells transfected with miR-203 mimics or mimic control were incubated with 5-FU at different concentrations, and 5-FU chemosensitivity was determined by MTT assay. ${ }^{*}$ p $<0.05$. Error bars represent the SD of samples in triplicate. 5-FU, 5-fluorouracil.

miR-203 overexpression increases 5-FU chemosensitivity in colorectal cancer cells. To further validate the role of miR-203 in the cellular response to 5-FU, we overexpressed miR-203 in LoVo/5-Fu (HR), Caco2 and HT29 cells by transient transfection with miR-203 mimics or mimic control, and miR-203 expression was confirmed by qRT-PCR assay (Fig. 3A). From the cytotoxicity assay, we found that miR-203 overexpression reduced the survival rate of $\mathrm{LoVO} / 5-\mathrm{Fu}(\mathrm{HR})$ cells compared with the controls (Fig. 3B). In line with the roles of miR-203 in LoVo/5-Fu (HR) cells, miR-203 overexpression resulted in a decrease in the survival rate of Caco2 cells (Fig. 3C) and HT29 cells (Fig. 3D). Taken together, these data suggest that miR-203 overexpression increases the cell sensitivity to 5-FU.

TYMS is a direct target of $m i R-203$. We discovered that there was a putative binding site for miR-203 in the 3'UTR of TYMS mRNA (Fig. 4A), which has been validated to be involved in 5-FU chemoresistance. We then constructed a luciferase reporter that contained the TYMS 3'UTR downstream of the luciferase coding gene. In addition, a mutant TYMS 3'UTR was also generated as shown in Fig. 4A. LoVo and Caco 2 cells were co-transfected with either miR-203 ASO or miR-203 mimics and wild-type or mutant TYMS 3'UTR for luciferase assay. As shown in Fig. 4B, we found that LoVo cells with miR-203 ASO had a higher level of TYMS 3'UTR intensity than the control cells, while $\mathrm{Caco} 2$ cells with miR-203 mimics had a lower intensity. However, neither miR-203 ASO nor miR-203 mimics affected the intensity of mutant TYMS
3'UTR (Fig. 4B). The luciferase assay suggests that miR-203 suppresses TYMS expression via binding to its 3'UTR. To further investigate the roles of miR-203 on TYMS protein level, colorectal cancer cells transfected with either miR-203 ASO or miR-203 mimics were subjected to western blot assay (Fig. 4C). We found that miR-203 inhibition increased TYMS protein levels, while miR-203 overexpression reduced TYMS expression. Overall, these data indicate that TYMS is a direct target of miR-203 in colorectal cancer cells.

Silencing of TYMS increases 5-FU chemosensitivity. Considering that TYMS is a target of miR-203, we next investigate the effects of TYMS on the cell sensitivity to 5 -FU in colorectal cancer cells. LoVo and Caco2 cells were transfected with TYMS-specific siRNAs and scrambled siRNA control. As shown in Fig. 5A, we found that cells with siRNA1 and siRNA2 specific for TYMS had a lower TYMS protein level than that in the control. Since siRNA2 resulted in a more marked decrease in TYMS level than siRNA1, we chose siRNA2 for the cytotoxicity assay. We found that TYMS knockdown suppressed the survival rate of the LoVo cells compared with the control (Fig. 5B). Similar data were obtained for $\mathrm{Caco} 2$ cells (Fig. 5C). These results indicate that TYMS knockdown increases cell sensitivity to 5-FU, similar to the roles of miR-203 overexpression.

miR-203 enhances 5-FU cytotoxicity in vivo. To determine whether the delivering of miR-203 in vivo increases the 
A

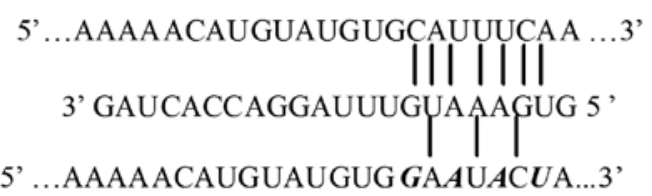

site 275-297 of TYMS 3'UTR

miR-203

mut TYMS 3'UTR

B

LoVo

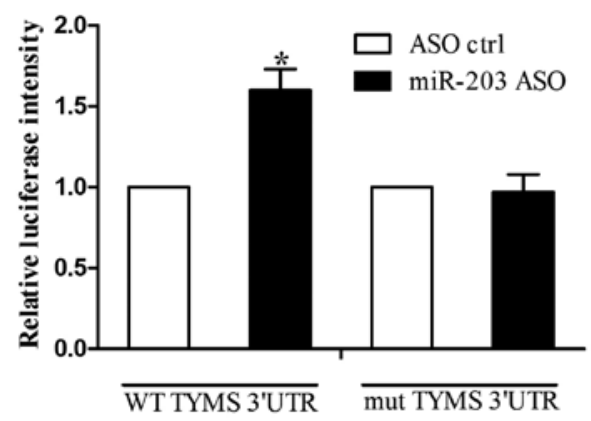

C

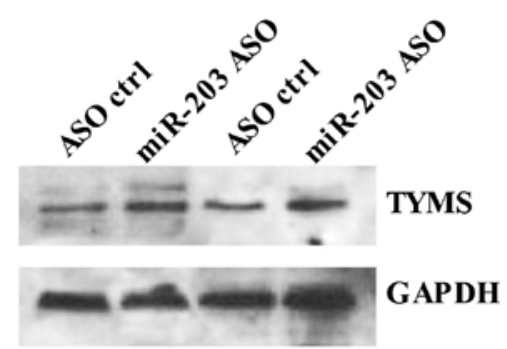

Caco2

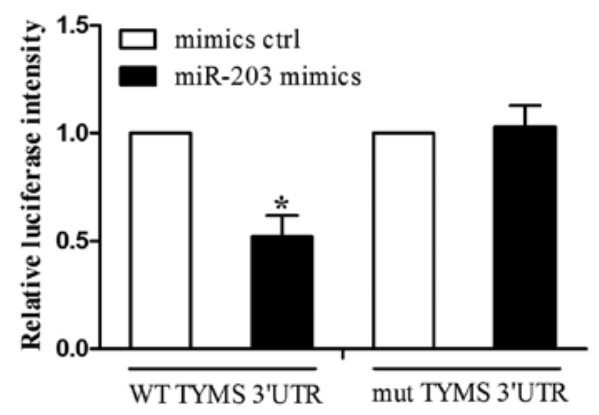

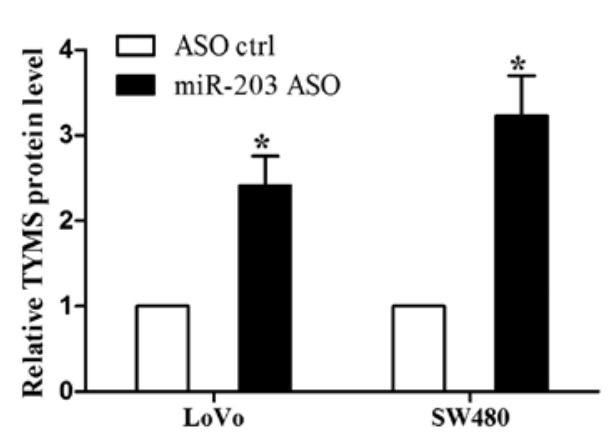

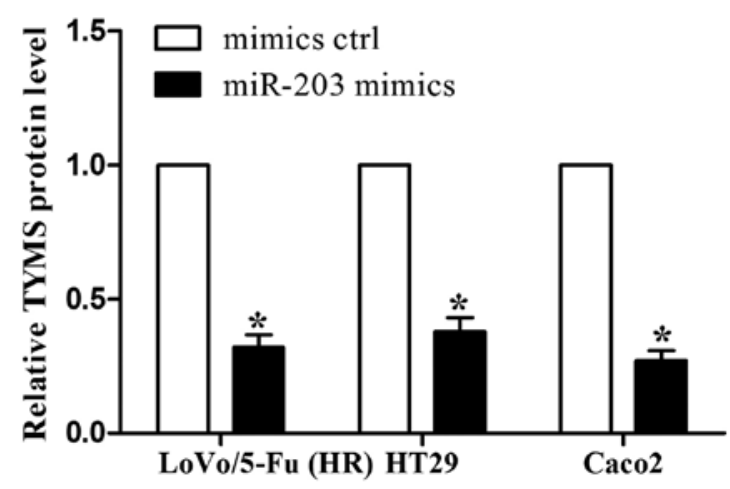

Figure 4. miR-203 negatively regulates TYMS expression via binding to its 3'UTR in colorectal cancer cells. (A) A putative binding site for miR-203 on the 3'UTR of TYMS mRNA is shown, and the mutation (bold and italic bases) within the binding site was generated. (B) Luciferase reporter assay was preformed to analyze the effect of miR-203 on the intensity of TYMS 3'UTR. LoVo and Caco2 cells were co-transfected with either miR-203 ASO or miR-203 mimics and wild-type or mutant TYMS 3'UTR, and luciferase intensity was determined. (C) Western blotting was performed to analyze the effect of miR-203 on TYMS protein levels. LoVo and SW480 cells transfected with miR-203 ASO or ASO control, and LoVo/5-Fu (HR), HT29 and Caco2 cells transfected with miR-203 mimics or mimic control were exposed to western blotting to analyze TYMS expression. GAPDH served as a loading control. * $\mathrm{p}<0.05$. Error bars represent the SD of samples in triplicate. TYMS, thymidylate synthase; 5-FU, 5-fluorouracil.

cytotoxic effect of 5-FU, we established a mouse colorectal cancer model by subcutaneously injected $10^{6}$ LoVo cells in NOD/SCID mice. When the volume of the tumor xenografts reached $\sim 100 \mathrm{~mm}^{3}$ (on day 12), we randomly separated the mice into four groups and each group was treated with either miR-203 control or miR-203, or 5-FU alone, or miR-203 together with 5-FU. The injection was performed every three days until day 25 when the tumors were collected. miR-203 expression was quantified by qRT-PCR in the tumor xenografts. We found that miR-203 expression increased in the mice treated with miR-203 (Fig. 6A), indicating that the delivery of miR-203 in vivo was successful. We then detected TYMS expression in the tumor tissues. As shown in Fig. 6B, the mice with miR-203 had a lower TYMS protein level than this level in the mice treated with miR-203 control or 5-FU alone. We also found that miR-203 alone did not affect tumor volume compared with the control. However, 5-FU treatment had a significant inhibitory effect on tumor growth. More importantly, when mice were treated with miR-203 and 5-FU together, tumor volume was inhibited more than with 5-FU treatment alone (Fig. 6C). The images of tumor xenografts are shown at day 25 in Fig. 6D. Taken together, these data suggest 
$\mathbf{A}$
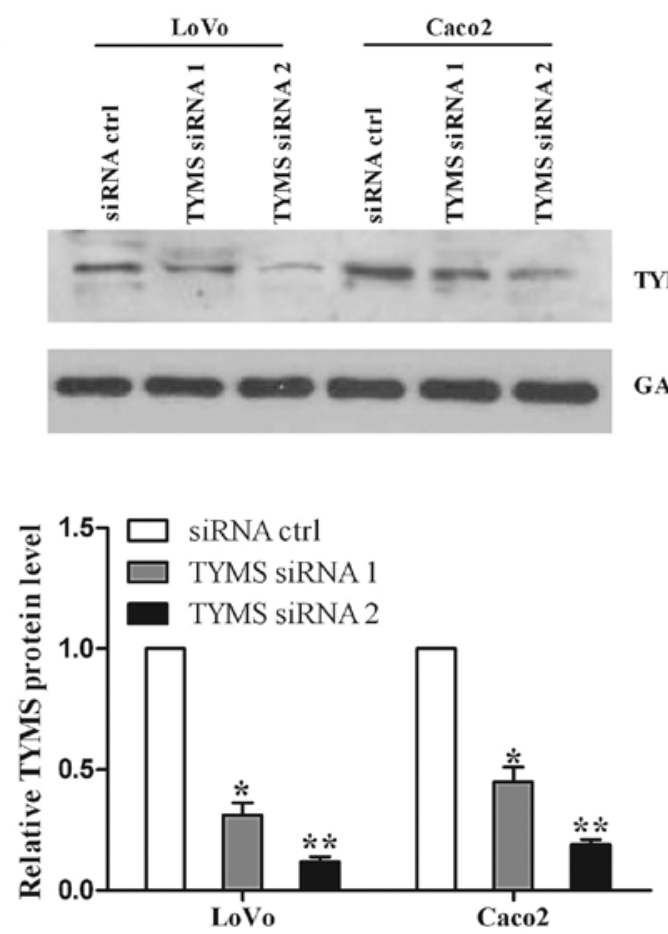

B

GAPDH

C
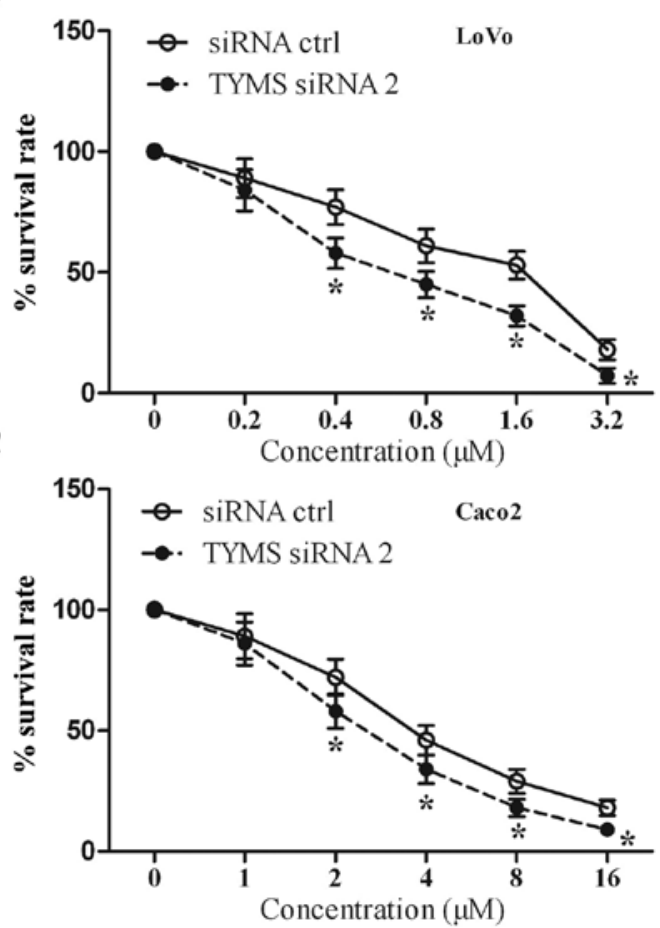

Figure 5. TYMS knockdown enhances the chemosensitivity to 5-FU in colorectal cancer cells. (A) LoVo and Caco2 cells transfected with siRNA specific for TYMS or scramble siRNA control were subjected to western blotting to analyze TYMS expression. GAPDH served as a loading control. (B and C) 5-FU chemosensitivity was determined by MTT in LoVo and Caco2 cells. LoVo and Caco2 cells transfected with TYMS siRNA or siRNA control were incubated with 5-FU at different concentrations, and cell viability was measured by MTT assay. " $\mathrm{p}<0.05$. ${ }^{* *} \mathrm{p}<0.01$. Error bars represent the SD of samples in triplicate. TYMS, thymidylate synthase; 5-FU, 5-fluorouracil.

A

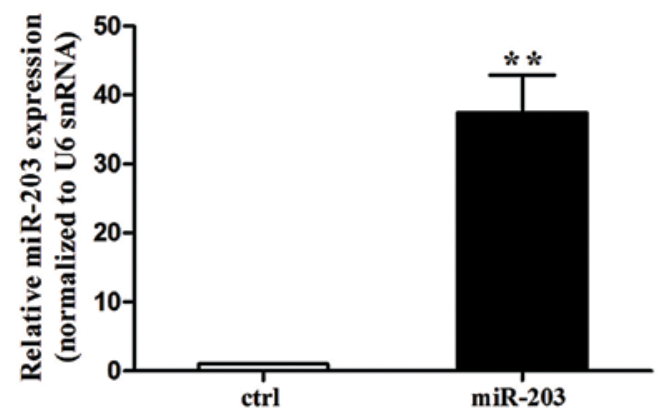

C

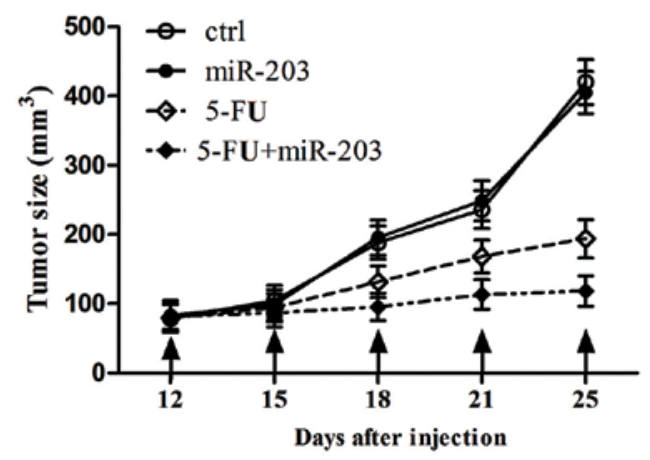

B

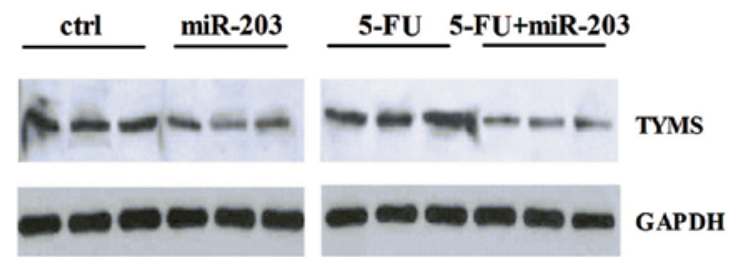

D

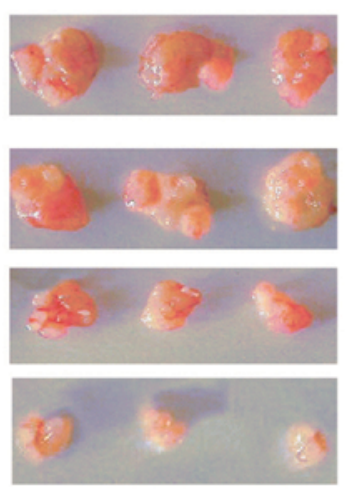

ctrl

miR-203

5-FU

5-Fu+miR-203

Figure 6. miR-203 enhances 5-FU cytotoxicity in vivo. (A) miR-203 expression was quantified by qRT-PCR in tumor xenografts and miR-203-injected tumors had a high miR-203 level. U6 snRNA served as an internal control for normalization.** $<<0.01$. Error bars represent the SD of samples in triplicate. (B) TYMS proteins levels were analyzed by western blotting in tumor xenografts that were treated with miR-203 precursor or miR-203 control or 5-FU or 5-FU in combination with miR-203. GAPDH served as a loading control. (C) NOD/SCID mice were injected with LoVo cells subcutaneously until day 12 when the tumor volume reached $\sim 100 \mathrm{~mm}^{3}$. On day 12, 15, 18, 21 and 25, mice were injected with miR-203 precursor or miR-203 control or 5-FU or 5-FU and miR-203 together, and the tumor volume was measured during treatments until day 25 when the mice were sacrificed. (D) Representative images of tumor xenografts are shown when mice were sacrificed at day 25. 5-FU, 5-fluorouracil; TYMS, thymidylate synthase. 
that miR-203 enhances the inhibitory effect of 5-FU on tumor growth via the downregulation of TYMS.

\section{Discussion}

Resistance to chemotherapy is one of the primary obstacles to effective cancer treatment (16), yet the mechanism is poorly clear in numerous types of cancers. In the present study, we demonstrated the molecular mechanism of 5-FU resistance in colorectal cancer cells. We found that miR-203 was downregulated in 5-FU-resistant $\mathrm{LoVo} / 5$-Fu cells compared with LoVo cells, and there was an inverse correlation between miR-203 and 5-FU resistance. miR-203 inhibition enhanced 5-FU chemoresistance, in contrast, miR-203 overexpression increased the sensitivity to 5-FU in colorectal cancer cells. Furthermore, TYMS was identified as a target of miR-203, and miR-203 suppressed the TYMS protein level. Silencing of TYMS had similar effects on 5-FU chemoresistance as that of miR-203 overexpression. Importantly, the inhibitory effect of 5-FU on tumor growth was enhanced by miR-203 in vivo.

miR-203 suppresses colon cancer cell proliferation and induces cell apoptosis, as well as increases the sensitivity to paclitaxel by downregulating Akt2 and its downstream genes that are involved in chemoresistance (17). miR-203 also reverses the chemoresistance to 5-FU by targeting Bmil in breast cancer cells (18). In addition, miR-203 suppresses cell proliferation, migration and invasion in prostate cancer (19), indicating that miR-203 plays tumor-suppressive roles. In line with the findings in previous studies, our data showed that miR-203 increased colorectal cancer cell sensitivity to 5-FU in vitro and in vivo via the suppression of TYMS. Surprisingly, miR-203 was reported to induce resistance to oxaliplatin by downregulating ATM kinase in colorectal cancer cells (20). Oxaliplatin causes cell apoptosis by inducing DNA damage, while paclitaxel plays antitumor roles by inducing $M$ phaseassociated apoptosis (21), and the action of 5-FU mainly depends on the interference of DNA synthesis. Different targets of miR-203 may be responsible for the cytotoxicity effects of oxaliplatin, paclitaxel and 5-FU on cancer cells, resulting in the different roles of miR-203 in chemotherapy. Previous studies indicate that miR-203 acts as a tumor-suppressor gene $(19,22)$. However, in a tumor growth study, we found that miR-203 overexpression did not affect tumor growth without 5-FU treatment. Our experimental concentration of miR-203 (5 nM) was much lower than the miR-203 used in the previous report (miR-203 lentivirus) (22). It is possible that miR-203 does not function as a tumor suppressor at a lower concentration.

To investigate the molecular mechanism of miR-203 in the cellular response to 5-FU, we validated that TYMS is a direct target of miR-203. miR-203 inhibition increased the luciferase intensity of TYMS 3'UTR, while miR-203 overexpression resulted in opposite effects. However, the inhibitory roles of miR-203 in TYMS expression was abolished when the binding site for miR-203 on TYMS 3'UTR was mutated. Furthermore, the TYMS protein level was suppressed by miR-203. TYMS is a cytosolic enzyme that alters the methylation of dUMP to dTMP and subsequently affects DNA synthesis and repair (23). The inhibition of TYMS is considered as a primary mechanism of 5-FU action (24). Accumulating evidence shows that patients with low TYMS expression have a higher response to 5 -FU and overall survival $(6,25,26)$. In contrast, overexpression of TYMS results in cell resistance to 5-FU $(27,28)$. Accordingly, our functional experiment showed that silencing of TYMS increased cell sensitivity to 5-FU and mediated the inhibitory effects of miR-203 on tumor growth under 5-FU exposure. Therefore, TYMS can act as a predictive biomarker of the cellular response to 5-FU and a therapeutic target for 5-FU-based chemotherapy.

In conclusion, the present study indicates that miR-203 enhanced the cell resistance to 5-FU in vitro and in vivo via the downregulation of TYMS in colorectal cancer, and provides novel insight into the molecular mechanism of 5-FU resistance. The present study will facilitate the development of a therapeutic strategy for 5-FU-based chemotherapy.

\section{Acknowledgements}

The study was supported by a grant (no. 2012-WSZD068) from the 2012 Medical Research Mentoring Program of Qingdao City.

\section{References}

1. Coget J, Borrini F, Susman S and Sabourin JC: Colorectal carcinomas in 2013: the search for powerful prognostic markers is still on the go! Cancer Biomark 14: 145-150, 2014.

2. No authors listed: Efficacy of adjuvant fluorouracil and folinic acid in colon cancer. International Multicentre Pooled Analysis of Colon Cancer Trials (IMPACT) investigators. Lancet 345: 939-944, 1995.

3. González-Vallinas M, Molina S, Vicente G, et al: Antitumor effect of 5-fluorouracil is enhanced by rosemary extract in both drug sensitive and resistant colon cancer cells. Pharmacol Res 72: 61-68, 2013.

4. Etienne MC, Chazal M, Laurent-Puig P, et al: Prognostic value of tumoral thymidylate synthase and p53 in metastatic colorectal cancer patients receiving fluorouracil-based chemotherapy: phenotypic and genotypic analyses. J Clin Oncol 20: 2832-2843, 2002.

5. Copur S, Aiba K, Drake JC, Allegra CJ and Chu E: Thymidylate synthase gene amplification in human colon cancer cell lines resistant to 5-fluorouracil. Biochem Pharmacol 49: 1419-1426, 1995.

6. Johnston PG, Lenz HJ, Leichman CG, et al: Thymidylate synthase gene and protein expression correlate and are associated with response to 5-fluorouracil in human colorectal and gastric tumors. Cancer Res 55: 1407-1412, 1995.

7. Yeh KH, Shun CT, Chen CL, et al: High expression of thymidylate synthase is associated with the drug resistance of gastric carcinoma to high dose 5-fluorouracil-based systemic chemotherapy. Cancer 82: 1626-1631, 1998.

8. Zhang LY, Liu M, Li X and Tang H: miR-490-3p modulates cell growth and epithelial to mesenchymal transition of hepatocellular carcinoma cells by targeting endoplasmic reticulum-Golgi intermediate compartment protein 3 (ERGIC3). J Biol Chem 288: 4035-4047, 2013

9. Bartel DP: MicroRNAs: genomics, biogenesis, mechanism, and function. Cell 116: 281-297, 2004.

10. Ambros V: The functions of animal microRNAs. Nature 431: 350-355, 2004.

11. Fujita Y, Kojima K, Hamada N, et al: Effects of miR-34a on cell growth and chemoresistance in prostate cancer PC3 cells. Biochem Biophys Res Commun 377: 114-119, 2008.

12. Hwang JH, Voortman J, Giovannetti E, et al: Identification of microRNA-21 as a biomarker for chemoresistance and clinical outcome following adjuvant therapy in resectable pancreatic cancer. PLoS One 5: e10630, 2010.

13. Bitarte N, Bandres E, Boni V, et al: MicroRNA-451 is involved in the self-renewal, tumorigenicity, and chemoresistance of colorectal cancer stem cells. Stem Cells 29: 1661-1671, 2011.

14. Karaayvaz M, Zhai H and Ju J: miR-129 promotes apoptosis and enhances chemosensitivity to 5 -fluorouracil in colorectal cancer. Cell Death Dis 4: e659, 2013. 
15. Gotanda K, Hirota T, Matsumoto N and Ieiri I: MicroRNA-433 negatively regulates the expression of thymidylate synthase (TYMS) responsible for 5-fluorouracil sensitivity in HeLa cells. BMC Cancer 13: 369, 2013.

16. Djeu JY and Wei S: Clusterin and chemoresistance. Adv Cancer Res 105: 77-92, 2009

17. Li JA, Chen YX, Zhao JF, Kong FR and Zhang YD: miR-203 reverses chemoresistance in p53-mutated colon cancer cells through downregulation of Akt2 expression. Cancer Lett 304: 52-59, 2011.

18. Yin J, Zheng G, Jia X, et al: A Bmil-miRNAs cross-talk modulates chemotherapy response to 5 -fluorouracil in breast cancer cells. PLoS One 8: e73268, 2013.

19. Viticchie G, Lena AM, Latina A, et al: MiR-203 controls proliferation, migration and invasive potential of prostate cancer cell lines. Cell Cycle 10: 1121-1131, 2011.

20. Zhou Y, Wan G, Spizzo R, et al: miR-203 induces oxaliplatin resistance in colorectal cancer cells by negatively regulating ATM kinase. Mol Oncol 8: 83-92, 2014.

21. Jordan MA and Wilson L: Microtubules as a target for anticancer drugs. Nat Rev Cancer 4: 253-265, 2004.

22. Tian L, Li M, Ge J, et al: MiR-203 is downregulated in laryngeal squamous cell carcinoma and can suppress proliferation and induce apoptosis of tumours. Tumour Biol 35: 5953-5963, 2014.
23. Lehman NL: Future potential of thymidylate synthase inhibitors in cancer therapy. Expert Opin Investig Drugs 11: 1775-1787, 2002.

24. Houghton JA, Tillman DM and Harwood FG: Ratio of 2'-deoxyadenosine-5'-triphosphate/thymidine-5'-triphosphate influences the commitment of human colon carcinoma cells to thymineless death. Clin Cancer Res 1: 723-730, 1995.

25. Popat S, Matakidou A and Houlston RS: Thymidylate synthase expression and prognosis in colorectal cancer: a systematic review and meta-analysis. J Clin Oncol 22: 529-536, 2004.

26. Lenz HJ, Hayashi K, Salonga D, et al: p53 point mutations and thymidylate synthase messenger RNA levels in disseminated colorectal cancer: an analysis of response and survival. Clin Cancer Res 4: 1243-1250, 1998.

27. Cho YB, Chung HJ, Lee WY, et al: Relationship between TYMS and ERCC1 mRNA expression and in vitro chemosensitivity in colorectal cancer. Anticancer Res 31: 3843-3849, 2011.

28. Galbiatti AL, Caldas HC, Maniglia JV, Pavarino EC and GoloniBertollo EM: Gene expression profile of 5-fluorouracil metabolic enzymes in laryngeal cancer cell line: predictive parameters for response to 5-fluorouracil-based chemotherapy. Biomed Pharmacother 68: 515-519, 2014. 